\title{
Clinical value in IVF: cycle characteristics associated with gonadotropin dose change in IVF patients using low responder protocols
}

\author{
Abstract \\ Objective: To identify patient and cycle characteristics that influence the frequency of \\ monitoring events and gonadotropin dose change during follicular monitoring of an IVF \\ cycle using a low responder protocol. \\ Design: Retrospective cohort study \\ Settings: Academic medical center
}

Patients: Infertile women undergoing IVF using a low responder protocol (GnRH agonist Flare or GnRH antagonist)

Intervention: Retrospective chart review

Main Outcome Measures: Gonadotropin dose change, initiation of GnRH antagonist, and administration of hCG

Results: Gonadotropin dose change in low responders rarely occurs in the first five days of gonadotropin stimulation in an IVF cycle. There were no baseline patient characteristics associated with change in medication management. After three days of gonadotropin stimulation, serum estradiol level $>300 \mathrm{pg} / \mathrm{ml}$ predicted any change in medication management for the next three days with $100 \%$ and $92 \%$ sensitivity for the Flare and Antagonist protocols, respectively.

Conclusion: For patients receiving a low responder protocol, there are early cycle characteristics associated with change in gonadotropin dose during the cycle. Monitoring intensity can likely be modified according to these criteria to reduce the total number of interventions.

Keywords: clinical value, follicular monitoring, gonadotropin dosing, low responder protocols
Volume 2 Issue 2 - 2015

\author{
John C Petrozza,' Irene Souter,' Mitchell \\ Scott Rein,' Joseph O'Brien Doyle,' Janelle \\ Katie Moulder,' John Meeker² \\ 'Vincent Department of Obstetrics and Gynecology, Harvard \\ Medical School, USA \\ ${ }^{2}$ Department of Environmental Health Sciences, University of \\ Michigan School of Public Health, USA
}

Correspondence: John C Petrozza, Division of Reproductive Medicine and IVF,Vincent Department of Obstetrics and Gynecology, Massachusetts General Hospital, 55 Fruit Street Boston, Massachusetts, 02I I4, USA, Tel (6I7) 726-6942, Fax (6I7) 724-7530,Email jpetrozza@mgh.harvard.edu

Received: December 04, 2014 | Published: March 9, 2015
Abbreviations: $\mathrm{E}_{2}$, estradiol; $\mathrm{CD}$, cycle day; IVF, in vitro fertilisation; HCG, human chorionic gonadotropin.

\section{Introduction}

Careful monitoring of estradiol $\left(\mathrm{E}_{2}\right)$ levels and ultrasound characteristics during an IVF cycle is paramount to ensuring an adequate response to gonadotropin stimulation, while mitigating the risk of hyper-response. Through these monitoring interventions, gonadotropin dosing can be appropriately titrated, start of a $\mathrm{GnRH}$ antagonist properly timed, and hCG accurately administered. Clinical experience guides the frequent decisions around monitoring frequency and gonadotropin dose changes. Certain protocols, such as the GnRH-antagonist, will require more frequent monitoring in order to identify specific mid-cycle events. Appreciation of particular trends has led to creation of stimulation protocols that require less intensive monitoring. ${ }^{1,2}$ There are likely particular patient characteristics that would allow accurate prediction of monitoring intervals. Rather than monitoring on "regular" intervals, an objectively-designed monitoring pattern could reduce the number of interventions and deliver comparable clinical outcomes. A reduction in the number of interventions would impact a number of fixed and variable costs involved in the monitoring process. This study aims to explore patient and cycle characteristics that influence the frequency of monitoring events and gonadotropin dose change in patients using a low responder protocol. By identifying parameters associated with monitoring frequency and gonadotropin dose changes, we intend to develop a framework for best-practice approach.

\section{Materials and methods}

The study was approved by the Partners Healthcare Institutional Review Board. Data from all IVF cycles leading to oocyte retrieval was obtained (01/01/2009-12/312009). Only patients receiving a low responder protocol (either the follicular GnRH-agonist flare or GnRH-antagonist protocol) were included in this study. All patients had a baseline ultrasound performed on designated cycle day (CD) two or three. A follicular ultrasound and serum $\mathrm{E}_{2}$ were obtained at all subsequent monitoring visits, and gonadotropin dosing was adjusted, as needed. The second monitoring event was performed on CD6. hCG was administered with at least three dominant follicles $(>15 \mathrm{~mm})$ present and $\mathrm{E}_{2} \geq 600 \mathrm{pg} / \mathrm{ml}$.

Information was collected on baseline patient characteristics (age, day-3 FSH, BMI, antral follicle count, history of prior cycle) and the number of days in cycle. Data was collected on the CD of the ultrasound, number of follicles seen (total, and $\geq 14 \mathrm{~mm}$ ), $\mathrm{E}_{2}$ level, day of GnRH-antagonist initiation and hCG- administration, and on whether gonadotropin dose increased, decreased, or remained unchanged. 
A management change was defined as any change in gonadotropin dosing, initiation of the GnRH-antagonist, or administration of hCG. Subgroup analysis of the actual data was used to estimate variable measurements for modeling different GnRH-antagonist start criteria (fixed $\mathrm{E}_{2}$ or cycle day). Cost estimates were based on 2012 Medicare reimbursement rates for a follicular ultrasound (\$92.02) and serum $\mathrm{E}_{2}$ $(\$ 39.32) .^{3}$

Primary outcomes included: changes in gonadotropin dosing, GnRH-antagonist initiation, and hCG administration. Chi-square, $\mathrm{t}$-test and Wilcoxon rank-sum test were used as appropriate $(\mathrm{p} \leq 0.05$ was considered significant).

\section{Results}

Complete data was available for 3,871 ultrasounds from 689 IVF cycles in 393 patients during this time period (22\% flare, and $14 \%$ antagonist cycles).

\section{GnRH-agonist flare cycles}

Ninety-nine patients using the flare protocol had 760 ultrasounds in 146 cycles (5.2 \pm 0.9 ultrasounds/cycle [range: $3-8]$, average cycle duration: $11.3 \pm 1.8$ days [8-16]). Figure 1 shows the distribution of ultrasounds performed in flare cycles and how monitoring relates to management change. From CD6 to CD8, one management change occurred for every 9.9 monitoring events. There were 24 dose decreases during this interval, and on $\mathrm{CD} 8, \mathrm{hCG}$ was administered to 5 patients. Only one live birth occurred in these 20 unique cycles. There was no difference in baseline patient characteristics for those who did and did not have a management change during this period.

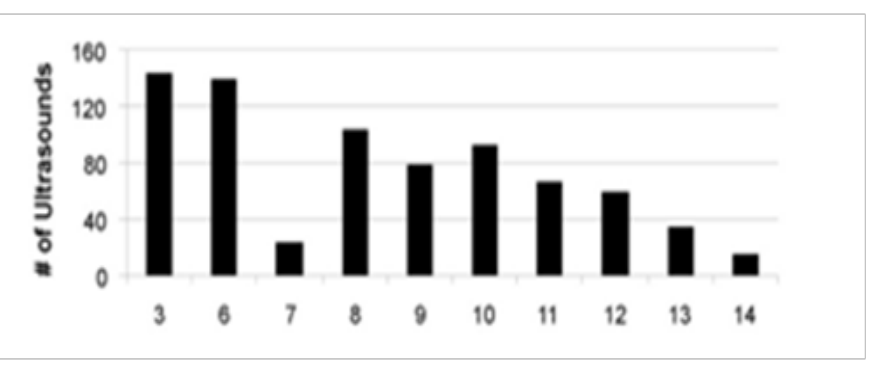

Figure I (A) Number of ultrasounds performed per cycle day.

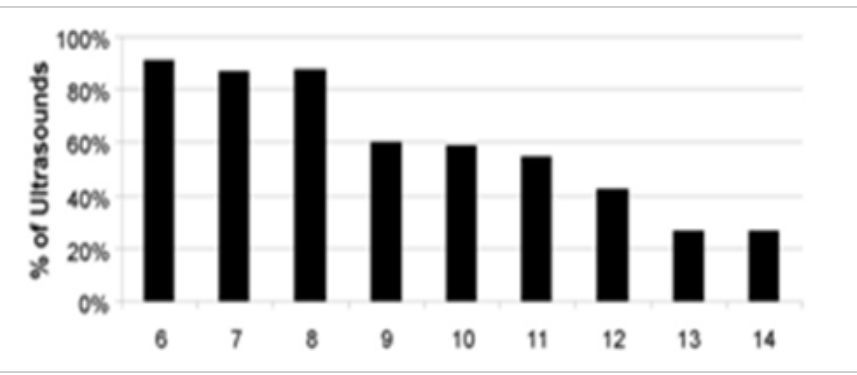

Figure I (B) Percent of ultrasounds by cycle day that do not result in a management change (gonadotropin dose change or administration of hCG).

Figure I GnRH agonist flare cycles.

A CD6 estradiol level of $300 \mathrm{pg} / \mathrm{ml}$ was $100 \%$ sensitive and $72.1 \%$ specific for identifying those who had a gonadotropin dose adjustment or hCG-administration during this time period. Of $138 \mathrm{CD} 6$ monitoring events, 51 cycles $(37.0 \%)$ had an $\mathrm{E}_{2} \geq 300 \mathrm{pg} / \mathrm{ml}$. Of those, 17 cycles $(33.3 \%)$ had a management change prior to CD9. No differences in baseline characteristics were identified between the groups that had a $\mathrm{CD} 6 \mathrm{E}_{2} \geq 300 \mathrm{pg} / \mathrm{ml}$ to those with lower $\mathrm{E}_{2}$ levels. Cycles with a CD6 $\mathrm{E}_{2}<300 \mathrm{pg} / \mathrm{ml}$ were significantly less likely to have a dose change anytime during their cycle compared to those with higher $\mathrm{E}_{2}$ levels $(\mathrm{p}<0.0001)$. Additionally, the higher estradiol population showed a greater average peak $\mathrm{E}_{2}$, and follicle number (total and $\geq 14 \mathrm{~mm}$ ) on the day of hCG-administration, and a lower average number of ultrasounds and day of hCG-administration (Table 1).

In patients with $\mathrm{CD} 6 \mathrm{E}_{2} \geq 300 \mathrm{pg} / \mathrm{ml}$, from 239 total monitoring events, there were 33 gonadotropin dose changes ( 29 decreases) in 22 unique cycles and individuals, giving an average of one gonadotropin dose change per 7.2 monitoring events. There was no difference in baseline patient characteristics or CD6 $\mathrm{E}_{2}$ level between those who did and did not have a management change. There was a significantly higher total number of follicles seen in cycles with a management change $(11.5 \pm 4.9$ versus $9.0 \pm 3.2, \mathrm{p}=0.05)$.

Table I Characteristics of $\mathrm{GnRH}$ agonist flare cycles according to cycle day six serum estradiol levels (mean \pm SD (range))

\begin{tabular}{|c|c|c|}
\hline & $\begin{array}{l}\text { Estradiol } \\
>300 \mathrm{ng} / \mathrm{ml}\end{array}$ & $\begin{array}{l}\text { Estradiol <300ng/ } \\
\mathrm{ml}\end{array}$ \\
\hline Peak Estradiol (ng/ml) & $\begin{array}{l}23 I I \pm 1 \mid 30(797- \\
5702)\end{array}$ & $|565 \pm 7| 3(374-38 \mid I)$ \\
\hline Total Number Follicles* & 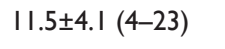 & $9.6 \pm 3.7(2-25)$ \\
\hline Number ultrasounds** & $4.8 \pm 0.8(3-7)$ & $5.5 \pm 0.9(4-8)$ \\
\hline HCG administration (CD) & $9.7 \pm 1.0(8-12)$ & $12.2 \pm 1.5(9-16)$ \\
\hline
\end{tabular}

*Denotes statistical significance at $\mathrm{p}=0.02$ level.

**Denotes statistical significance at $\mathrm{p}<0.00 \mathrm{I}$ level.

In patients with CD6 $\mathrm{E}_{2}$ levels $<300 \mathrm{pg} / \mathrm{ml}$, there were 473 monitoring events, with 20 gonadotropin dose changes (19 decreases, all after CD8) in 13 unique cycles and individuals. hCG was not administered in any of these cycles before $\mathrm{CD} 9$, (one gonadotropin dose change per 23.7 monitoring events). Gonadotropin dose change occurred on average on $\mathrm{CD}$ : $10.9 \pm 1.4$ (9-13). Those requiring a dose decrease had peak $\mathrm{E}_{2}$ levels: 2588 $\pm 893 \mathrm{pg} / \mathrm{ml}$ (1292-3811). Compared to all cycles with a day- $6 \mathrm{E}_{2}<300 \mathrm{pg} / \mathrm{ml}$, those requiring a dose decrease had a significantly higher number of follicles (total and $\geq 14 \mathrm{~mm}: 12.5 \pm 0.7$ vs. $9.8 \pm 0.4, p=0.01$, and $6.5 \pm 0.7$ vs. $4.7 \pm 0.3$, $\mathrm{p}=0.02$, respectively), although there was no significant difference in peak $\mathrm{E}_{2}$ levels.

\section{GnRH antagonist cycles}

Following a conventional monitoring approach, 555 ultrasounds were performed for 93 antagonist cycles in 76 unique individuals (6.0 \pm 1.0 ultrasounds/cycle [range: 4-9], average cycle duration: $11.6 \pm 1.6$ days [8-17]). The GnRH-antagonist was started on CD $9.0 \pm 1.6$ (6-15) with a mean $\mathrm{E}_{2}: 874 \pm 400 \mathrm{pg} / \mathrm{ml}$ (230-2019), (Table 2).

Clearly, a large number of ultrasounds were performed between CD6 and CD10 that did not result in gonadotropins dose changes in Figures 2(A \&B). Most of these ultrasounds were being performed to optimally time the GnRH-antagonist initiation, as indicated by the large number of antagonist starts from CD8 to CD10 (Figure 2C). From CD6 to $\mathrm{CD} 8$, one management change occurred for every 3.7 monitoring event; excluding antagonist starts, one management change occurred every 12.1 monitoring events. 
Table 2 Characteristics of GnRH antagonist cycles based on actual cycle data or subgroup analysis of actual data (mean \pm SD (range))

\begin{tabular}{|c|c|c|c|c|}
\hline & Actual & Fixed Estradiol & Fixed Cycle Day 8 & Fixed Cycle Day 9 \\
\hline Antagonist Start Day & $\begin{array}{l}9.0 \pm 1.6 \\
(6-15)\end{array}$ & $\begin{array}{l}10.1 \pm 1.9^{* * *} \\
(6-15)\end{array}$ & - & - \\
\hline Estradiol level (ng/ml) & $\begin{array}{l}874 \pm 400 \\
(230-2019)\end{array}$ & $\begin{array}{l}1 \mid 44 \pm 236 * * \\
(448-1915)\end{array}$ & $\begin{array}{l}715 \pm 482^{* *} \\
(92-2359)\end{array}$ & $\begin{array}{l}1051 \pm 592 \\
(36-2610)\end{array}$ \\
\hline Follicles $\geq 14 \mathrm{~mm}$ & $\begin{array}{l}0.9 \pm 1.4 \\
(0-6)\end{array}$ & $\begin{array}{l}2.8 \pm 2.6^{\text {*k }} \\
(0-12)\end{array}$ & $\begin{array}{l}0.6 \pm 1.2^{*} \\
(0-5)\end{array}$ & $\begin{array}{l}2.3 \pm 2.7^{\text {** }} \\
(0-12)\end{array}$ \\
\hline
\end{tabular}

*Denotes statistical significance at $\mathrm{p}=0.04$ level, relative to actual levels.

Denotes statistical significance at $p<0.00$ I level, relative to actual levels.

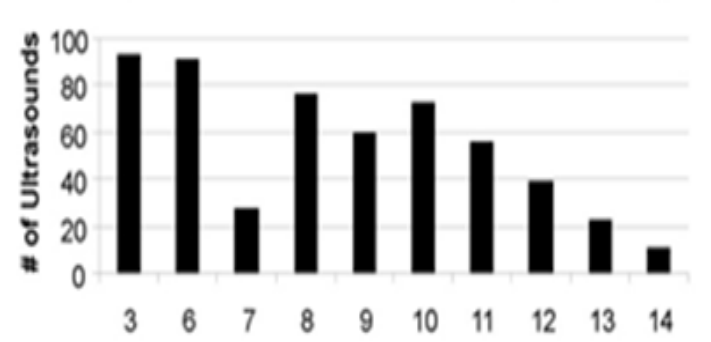

Figure 2 (A) Number of ultrasounds performed by cycle day.

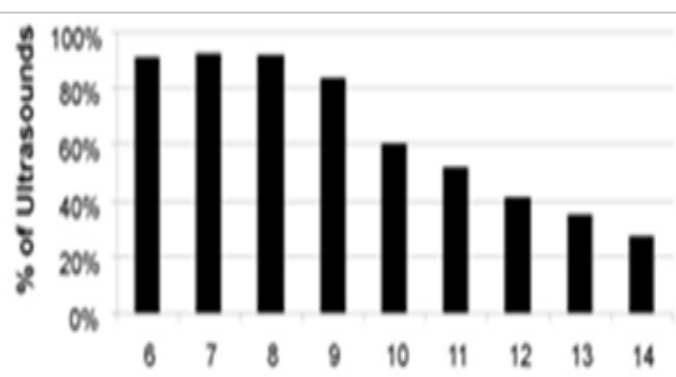

Figure 2 (B) Percent of ultrasounds by cycle day that do not result in a management change (gonadotropin dose change or administration of hCG).

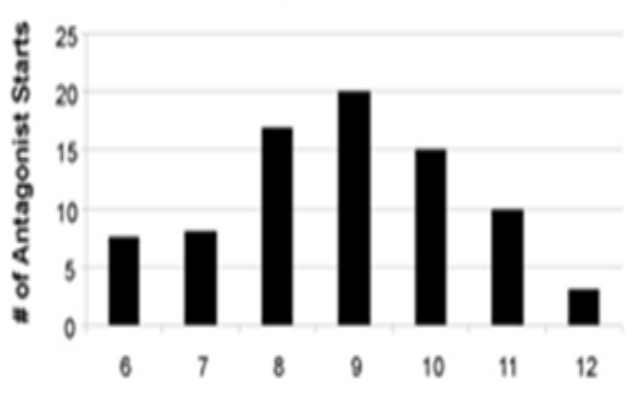

Figure 2 (C) Number of antagonists starts by cycle day.

Figure $2 \mathrm{GnRH}$ antagonist cycles.

Using a fixed cycle day GnRH-antagonist start provided a closer and more conservative fit than using a fixed $\mathrm{E}_{2}$ level in terms of mimicking conventional monitoring. Statistical comparisons between antagonist start day, serum $\mathrm{E}_{2}$ levels, and the number of follicles $\geq 14 \mathrm{~mm}$ were made between the cycles based on the actual and predicted antagonist start day (Table 2). The fixed $\mathrm{E}_{2}$ level start at $1000 \mathrm{pg} / \mathrm{ml}$ produced a significantly later antagonist start in the cycle at a higher $\mathrm{E}_{2}$ level with more follicles $\geq 14 \mathrm{~mm}$.

For the fixed GnRH antagonist cycle day start, comparisons were made between a fixed CD8 or CD9 start, and the actual monitoring data (Table 2). Data from a CD9 start day showed a similar $E_{2}$ level at initiation of the GnRH-antagonist, but significantly more follicles $\geq 14 \mathrm{~mm}$. A CD8 start was more optimal from a conservative perspective: both $\mathrm{E}_{2}$ levels and number of lead follicles were less than that observed in the actual data. Several details require additional consideration if using a fixed CD8 GnRH-antagonist start. First is the number of cycles that had a GnRH-antagonist start before CD8 (15/93 or $16 \%)$. $\mathrm{E}_{2}$ level on the GnRH- ntagonist start day for these fifteen cycles was $640 \pm 280 \mathrm{pg} / \mathrm{ml}$ (range: $242-1271$ ) with $0.2 \pm 0.4$ follicles $\geq 14 \mathrm{~mm}$ (one cycle resulted in a live birth, three in biochemical conceptions and eleven in no pregnancy).

Secondly, we analyzed how often gonadotropin dose changes being made using intensive monitoring would be missed if monitoring was not resumed until after the GnRH-antagonist start. Eight unique individuals $(8.6 \%)$ had at least one gonadotropin dose change prior to $\mathrm{CD} 9$, seven of which were a gonadotropin decrease on CD6 (peak $\mathrm{E}_{2}: 3003 \pm 882 \mathrm{pg} / \mathrm{ml}$ ). Lastly, we analyzed how timing of hCGadministration would be impacted if monitoring did not resume until CD9. Two cycles had hCG administered on CD8 without prior dose changing (peak $\mathrm{E}_{2}: 990$ and $2359 \mathrm{pg} / \mathrm{ml}$, with 3 and 5 follicles $\geq 14 \mathrm{~mm}$, resulting in a biochemical pregnancy and a negative $\mathrm{hCG}$, respectively).

A CD6 serum $\mathrm{E}_{2}$ could potentially identify patients undergoing rapid, early recruitment or greater than expected response to gonadotropins. Using an $\mathrm{E}_{2} \geq 300 \mathrm{pg} / \mathrm{ml}$ would have identified 30 patients requiring further evaluation. All eight patients who had a gonadotropin dose change and both patients undergoing hCGadministration prior to CD9 were in this population. Thirteen of 15 patients who initiated the antagonist prior to $\mathrm{CD} 8$ were included by these criteria. However, one of the missed cycles resulted in live birth. This serum $\mathrm{E}_{2} \geq 300 \mathrm{pg} / \mathrm{ml}$ would provide a sensitivity and specificity for identifying a change in management of $92.0 \%$ and $89.7 \%$. There were no differences in baseline patient characteristics for those who did and did not have a management change when $\mathrm{E}_{2}$ was $\geq 300 \mathrm{pg} / \mathrm{ml}$.

\section{Discussion}

There is a cost that influences the total expense of an IVF cycle. Direct costs include staffing, space, supplies, laboratory expense, 
and amortization of ultrasound and equipment. Indirect costs include patient time (away from their work) and travel. In a future foreshadowed by detailed scrutiny of clinical value in healthcare, these are certainly important considerations. Anticipating them will allow tailoring of monitoring regimens, such that they continue to deliver robust clinical outcomes at optimized cost.

Clinical value is a metric of increasing interest in this era of cost reform. ${ }^{4-11}$ This study has focused on this issue of reducing the cost of care delivery, while maintaining comparable clinical outcomes. Just as careful attention is directed towards protocol selection, the monitoring regimen can be similarly considered. For the optimal approach, monitoring intensity/cost can be modified according to the number of serum hormone measurements and number ultrasounds.

Knowing of patient characteristics that influence the frequency of monitoring and likelihood of dose change will allow the stratification of patients into monitoring regimens of varying intensities. We were not able to identify a priori patient characteristics associated with changes in management in flare or antagonist cycles. As an alternative to baseline characteristics, early events in a stimulated cycle can be also used to stratify monitoring intensity. Low responders have the appeal of reduced variability in gonadotropin dosing compared to the general IVF population.

Flare cycles had a large number of ultrasounds performed from CD6 to CD8, with rare changes in management. As only one live birth resulted from 27 management changes in 20 unique cycles (5\%), it is debatable whether it is necessary to isolate these cycles. Nevertheless, using a estradiol threshold of $300 \mathrm{pg} / \mathrm{ml}$ on CD6 is a practical alternative, as there is a large difference in ratios of monitoring events that are performed per management change (7.2 events for $E_{2}$ $>300 \mathrm{pg} / \mathrm{ml}$ vs. 23.7 events for lower levels). Additionally, a CD6 $\mathrm{E}_{2}$ $\geq 300 \mathrm{pg} / \mathrm{ml}$ provided $100 \%$ sensitivity in identifying cycles in which management changes occurred prior to CD9.

A potentially more cost effective consideration would be to administer hCG solely according to follicular measurement parameters, without the use of serum $\mathrm{E}_{2}$. Within the overall population of CD6 $\mathrm{E}_{2}$ $<300 \mathrm{pg} / \mathrm{ml}$, those that did require a dose change were compared to those that did not. A gonadotropin step-down would be an appropriate consideration in the terminal portion of stimulation with numerous follicles ( $\geq 8$ total follicles, based on the minimum range in the group with later management changes).

The above data suggest a method whereby the flare population is stratified according to CD6 $\mathrm{E}_{2}$ level. Using the assumptions that those with $\mathrm{E}_{2}>300 \mathrm{pg} / \mathrm{ml}$ maintain the same monitoring intensity, while those with lower levels would forgo additional monitoring until CD9, at which point they would resume the original monitoring intensity, this approach could eliminate 136 ultrasounds (29.4\%) and $56 \mathrm{E}_{2}-$ measurements, amounting to a reduction in cost of $\$ 14,716.64$. Figure 3 illustrates our proposed flare monitoring protocol.

For antagonist protocols, we currently use two criteria to start the GnRH-antagonist: serum $\mathrm{E}_{2}$ level or a lead follicle size. Monitoring events were occurring in these cycles to adjust gonadotropin dosing, accurately time the start of the GnRH-antagonist, and administer hCG. Between CD6 and CD8, one of these three events occurred for every 7.7 monitoring episodes. Using a fixed level of $1000 \mathrm{pg} / \mathrm{ml}$ provided a poor fit, as it initiated the antagonist significantly later than with the conventional approach, which could predispose the cycle to either escape ovulation or early luteinization. A lower $\mathrm{E}_{2}$ threshold (900pg/ $\mathrm{ml}$ ), may be an alternative to consider.

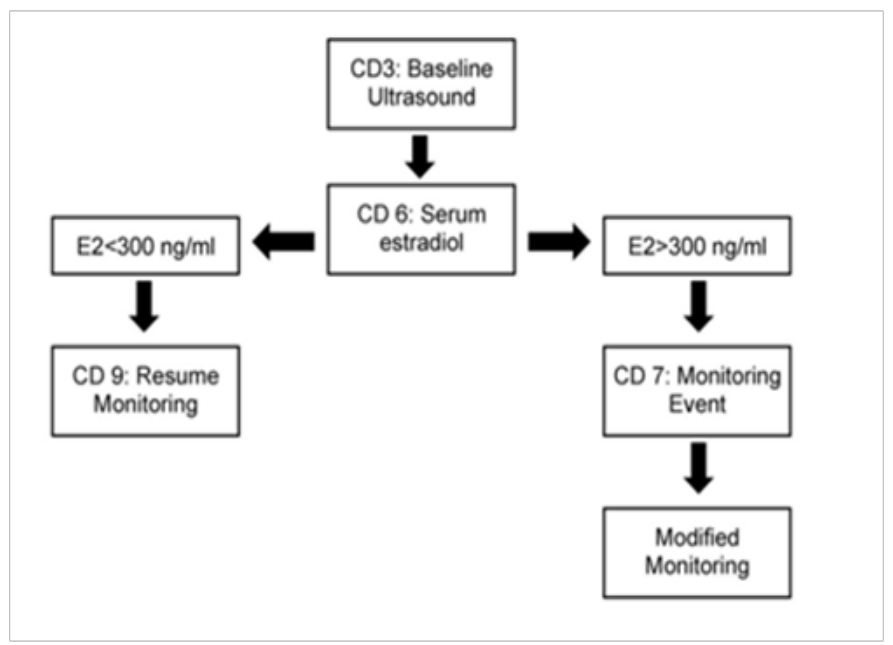

Figure 3 Proposed GnRH agonist flare monitoring algorithm.

$26.8 \%$ of the cycles had a management change prior to CD9, and it may be important to capture this segment of the population, although they often reflect an effort to salvage cycles with rapid recruitment of a small number of follicles. To address these concerns, it is reasonable to obtain a CD6 $\mathrm{E}_{2}$ level, using $300 \mathrm{pg} / \mathrm{ml}$ as a threshold indicator for additional monitoring. Using this cutoff, $32 \%$ of cycles would require additional monitoring, and there would be accurate identification of management changes. Figure 4 illustrates our proposed antagonist monitoring algorithm. A fixed $\mathrm{CD} 8 \mathrm{GnRH}$-antagonist start is appealing in that it requires less intensive monitoring, closely approximates the actual data, and represents a more conservative approach (Table 2).

Eliminating all monitoring from the baseline until after CD8 would result in the exclusion of $194(35.0 \%)$ ultrasounds, and the same number of $\mathrm{E}_{2}$ measurements. Conservatively, if ultrasound monitoring and $\mathrm{E}_{2}$ measurements proceeded according to conventional monitoring pattern, this would result in a reduction in cost of $\$ 25,479.96$.

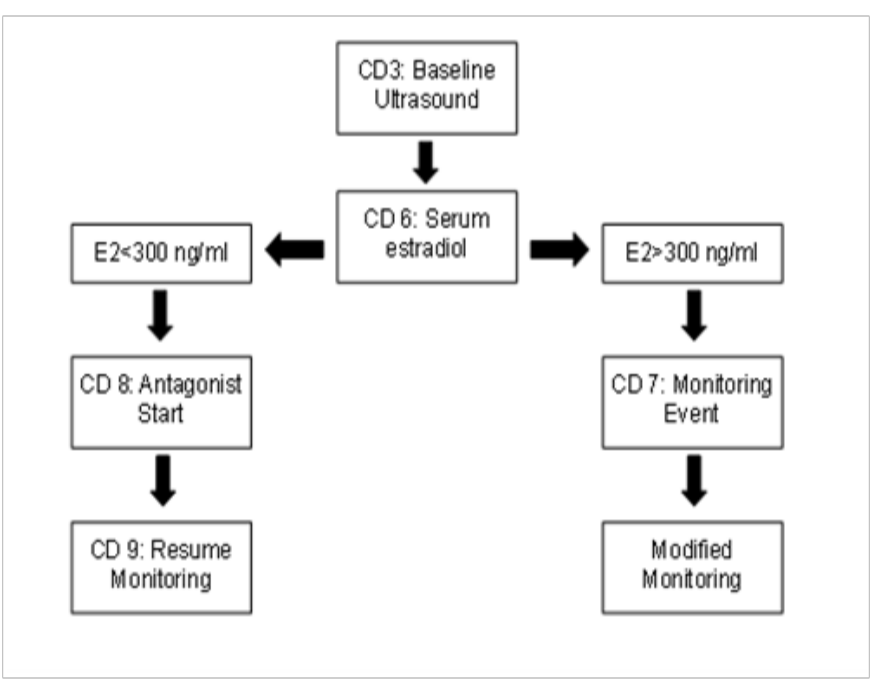

Figure 4 Proposed GnRH antagonist monitoring algorithm. 
Although reduced costs by these algorithms are static assessments, spin-off benefits would likely include improved patient satisfaction and distribution of resources. Optimistically, downstream monitoring density may also be reduced. Not included in this modeling is the cost of failed cycles using the new system, if it proves suboptimal. At a cost of approximately $\$ 10,000$ per IVF cycle, a small number of failed cycles would quickly off-set the cost-savings of a more efficient stimulation protocol..$^{12}$ Prospective monitoring would elucidate how these methods perform in their ability to reduce costs and maintain clinical outcomes.

Caution is appropriate in application of these methods. Our population was specifically low responders. Further, these data reflect a uniform approach used within a single center, and there is considerable variability between centers in gonadotropin dosing, and the tolerance of $\mathrm{E}_{2}$ levels and number of recruited follicles.

The aim of this study is to identify methods of limiting overutilization, which increases costs without improving outcomes. Often, IVF monitoring develops from a combination of experience and habit, although this can potentially be streamlined according to a process and outcomes-driven approach. Multiple other areas within medicine, previously considered too inherently variable, have already been successfully converted into best-approach models. Though achieving exceptional success rates is the foremost concern, optimizing workflow and resource-allocation will be a necessity for the economic viability of the healthcare system and all practices within it, regardless of size. Many indicators predict that the practices most effective at accomplishing this will harness a competitive advantage going forward. ${ }^{7-10}$

\section{Acknowledgments}

None.

\section{Conflicts of interest}

The authors declare there is no conflict of interests.

\section{References}

1. Hurst BS, Tucker KE, Awoniyi CA, et al. Preprogrammed, unmonitored ovarian stimulation reduces expense without compromising the outcome of assisted reproduction. Fertil Steril. 1997;68(2):282-286.

2. Hurst BS, Tucker KE, Schlaff WD. A minimally monitored assisted reproduction stimulation protocol reduces cost without compromising success. Fertil Steril. 2002;77(1):98-100.

3. Centers for Medicare and Medicaid Services. Medicare Allowable Fee Schedule. 2012

4. Bates DW, Spell N, Cullen DJ, et al. The costs of adverse drug events in hospitalized patients. Adverse drug events prevention study group. JAMA. 1997;277(4):307-311.

5. Kohn LT. Organizing and managing care in a changing health system. Health Services Research. 2000;35(1 Pt 1):37-52.

6. McGlynn EA, Asch SM, Adams J, et al. The quality of health care delivered to adults in the United States. N Engl J Med. 2003;348(26):2635-2645.

7. Porter ME. Value-based health care delivery. Ann Surg. 2008;248(4):503509.

8. Porter ME. A strategy for health care reform--toward a value-based system. N Engl J Med. 2009;361(2):109-112.

9. Porter ME. What is value in health care? NEnglJMed.2010;363(26):2477_ 2481.

10. Porter ME, Teisberg EO. Redefining competition in health care. Harv Bus Rev. 2004;82(6):64-76.

11. Porter ME, Teisberg EO. How physicians can change the future of health care. JAMA. 2007;297(10):1103-1111.

12. Hirshfeld-Cytron J, Grobman WA, Milad MP. Fertility preservation for social indications: a cost-based decision analysis. Fertil Steril. 2012;97(3):665-670. 